\title{
Edge Detection Using Fuzzy Double Gradient Morphology
}

\author{
Dillip Ranjan Nayak
}

\begin{abstract}
Detecting the edges of objects within images is a critical task for quality image processing. This paper proposes an edge detection operator based on the combination of fuzzy gradient morphology and Sobel operator. When we use traditional detection operators to detect the edge of an object in an image, we get lots of noisy Points. In this paper, we demonstrate a technique in which we preprocess an image with Sobel operator and then apply gradient morphology. This method effectively removes the noise and gives good detail image edge detection. We evaluate the method quantitatively and compare it to classical morphological method. Our fuzzy based edge segmentation method performs better than the classical edge detectors. Since the proposed methods are based on fuzzy morphological operations, these are efficient and enhanced.
\end{abstract}

Keywords--- Fuzzy, Mathematical Morphology, Segmentation, Sobel Operator

\section{INTRODUCTION}

$\mathrm{T}$ ODAY edge detection is a very important area in the field of image processing and computer vision. It also plays an important role for image segmentation and object recognition. Edge detection is the process to detect the important features of image. Here, features mean the properties of image like discontinuities in physical and geometric characteristics of an image or abrupt variation in its intensity, Wang et al.,[1]. The quality of edges is affected by the presence of objects in similar illumination, noise and density of edges,Nadarnejat [14]. The variation in characteristics can lead to the variation in gray level of the image. Edge detection is therefore considered as an important step for facilitating higher level image analysis and processing, Mittal and Batra [2] and Gonzalez et al., [3].

Conventionally, edge was detected using gradient algorithms like Sobel, Prewitt andLaplacian of Gaussian operator,Huertas and Medioni [4], all of which belong to the high pass filtering methods. Another important gradient based edge detection method is Canny algorithm which solves an optimization problem to detect the edges, Canny [5]. The tradeoff between detection and location of edge pixels make a problem in accuracy,Wang et al., [1]. By changing threshold values, edge detection rate increases, but the accuracy of edge

Dillip Ranjan Nayak, Asst. Professor, Computer Science \& Engineering, Govt. College of Engineering, Kalahandi, Bhawanipatna , Odisha, India. Email:dillip678in@yahoo.co.in

DOI: 10.9756/BIJAIP.10357 location decreases. Because of noise, low contrast and some other factors, edge detection methods cannot give satisfactory results, Marr and Hildreth [6]. There are some edge detection algorithms in frequency domain, Musevi-Niya andAghagolzadeh[7]where over or under edge based segmentation occurs.

As the performance of classical edge detectors degrades with noise, morphological edge detector has been studied, Lee et al., [8].It was introduced by Matheron as a technique for analyzing geometric structure of metallic solids. It was extended to image analysis,Serra [9].Mathematical morphology is a new mathematical theory which can be used to process and analyze the images, Maragos [10],Richard [11] and Jean [12]. It provides an alternative approach to image processing based on shape,concept stemmed from set theory, Serra [9]. In the mathematical morphology theory, images are treated as sets, and morphological transformations which are derived from Minkowski addition and subtraction are defined to extract features in images. A fundamental advantage of mathematical morphology applied to image is that it is intuitive since it works directly on spatial domain.

The idea of fuzzy logic is to extend the binary (TRUE or FALSE) computer model with some uncertainty or blur.Many of our sensory impressions are qualitative and imprecise and, therefore, unsuitable for accurate measurements. For example, a pixel is perceived as "dark", "bright" or even "very bright", but not as pixels with the gray scale value "231". Fuzzy quantities are based mathematically on the fuzzy set theory, in which the belongingness of an element to a set of elements is not restricted to the absolute states TRUE (1) or FALSE (0), but continuously defined within the entire interval [0..1].

In general mathematical morphology, operation definitions are similar to set theory and set operation definitions. For this reason fuzzy set theory is easily applied to the mathematical morphology, Nadarnejat [15]. Mathematical morphology is a collection of operations which produces useful outcomes in image processing area. It is completely based on set theory. For this reason all of the operations in morphology are defined on the simple set operation rules to apply them on image pixels. The basic mathematical morphological operators are dilation and erosion and the other morphological operations are the synthesization of the two basic operations.The objective of this paper is to present the hybrid approach for edge detection. Under this technique, edge detection is performed in two phases. In first phase, sobel algorithm is applied for image smoothing and in second phase fuzzy morphology is applied to detect actual edges. Fuzzy morphology is a wonderful tool for edge detection. Fuzzy mathematical morphology is beneficial for detection of the 
edges of a variety of geometrical shapes along with the opening and closing filtering.The proposed method improves the visibility of edges of images.

Section 1 introduces the concept of different methods used for edge detection. Morphological Image processing is explained in Section 2. Section 3 presents the proposed algorithm in detail. Then, this method is tested in section 4 using rice image, and its performance is compared with that of traditional method. Finally, Section 5 illustrates some concluding remarks.

\section{MORPHOLOGICAL IMAgE PROCESSING}

Mathematical morphology is a non -linear approach for detection of edges. Mathematical morphology is a tool for extracting image components that are useful in representation and description of region shape, such as boundaries, skeletons and convex hull. Morphology is a mathematical framework for the analysis of spatial structures which are totally based on set theory. It is a strong tool for performing many image processing applications. Morphological sets represent important values. Mathematical morphology is used to study geometric structure of images, Zhaoet al., [17] and Zhao et al., [18]. The basic idea above is to apply a structuring element to detect an image, to see whether the structuring element can be filled in the internal of the image well and to validate the method. The important morphological operations are basically dilation, erosion, open and close operations. Morphological operations make use of a structuring element $\mathrm{M}$; which can be either a set or a function that corresponds to a neighborhoodfunction related to the image function $\mathrm{g}(\mathrm{x})$, Soille [13]. In general, a dilation (denoted by $\bigoplus$ ) is a mathematical operator that commutes with the supremum operation. On the other hand, erosion (denoted by $\Theta$ ) is a mathematical operator that commutes with the infimum operation. There is a homomorphism between the image function $\mathrm{g}$ and the set $\mathrm{B}$ of all pixels with image function value 1 . The structuring element $\mathrm{M}(\mathrm{x})$ is a function that assigns a subset of $\mathrm{N} \times \mathrm{N}$ to every pixel of the image function. Then, dilation, an increasing transformation, is defined as

$$
\mathrm{B} \oplus \mathrm{M}=\mathrm{U} \mathrm{x} \in \mathrm{B} \mathrm{M}(\mathrm{x})
$$

Whereas, erosion, a decreasing transformation, is defined as

$$
\mathrm{B} \ominus \mathrm{M}=\{\mathrm{x} \mid \mathrm{M}(\mathrm{x}) \subseteq \mathrm{B}\}
$$

In the same manner, opening and closing of set $\mathrm{B}$ by structuring element $M$ are respectively defined as

$$
\begin{gathered}
B \circ M=((B \ominus M) \oplus M), \\
\text { And } B \cdot M=((B \oplus M) \ominus M) .
\end{gathered}
$$

\section{PROPOSED WORK}

First we find edges using Morphological edge detection algorithm which selects appropriate structuring element of the processed image and makes use of the basic theory of morphology including erosion, dilation, operation and the synthesization operations on them to get clear image edge. In the process, the synthesized modes of the operations and the feature of the structuring element decide the result of the processed image. In edge detection, we must select appropriate structuring element by a $3 \times 3$ square. StructuringElements are used in morphological theory, which are also represented as matrices. Structuring element is a characteristic of certain structures and features to measure the shape of an image and is used to carry out other image processing operations. The shape and size of the structuring element (SE)plays a crucial role in image processing and is therefore chosen according to the condition of the image and demand of processing.

The dilation and erosion often are used to compute the morphological gradient of image $B$, denoted by $G(B)$ : $G(B)=$ $(\mathrm{M} \oplus \mathrm{B})$ - $(\mathrm{M} \ominus \mathrm{B})$. The effect of erosion and dilation operations is better forimage edge which can be verified by performing the difference between processed image and original image, but they are worse for noise filtering. So we modify this work using hybrid method, combination of fuzzy and Sobelalgorithm. Sobel Gradient is widely used in image processing techniques. The Sobel kernels are more suitable to detect edges along the horizontal (180 degree) and vertical axis (90 degree), Kekre et al., [16]. The Sobel operator is based on convolving the image with a small, separable, and integer valued filter.Using sobel the edges of images are in under segmentation. So in this paper fuzzification has done to avoid undersegmentation and enhance the image.

Fuzzy image processing has three main stages: image fuzzification, modification of membership values, and, if necessary, image defuzzification. The fuzzification and defuzzification steps are due to the fact that we do not possess fuzzy hardware. Therefore, the coding of image data (fuzzification) and decoding of the results (defuzzification) are steps that make possible to process images with fuzzy techniques

Our proposed algorithm as follows.

1. Read original image $B$

2. Find gradient image $G(B)$ using Sobel operator .

3. Fuzzify gradient image using membership function $\mathrm{r}=\frac{\mathrm{d}-\mathrm{mn}}{\mathrm{mx}-\mathrm{mn}}$ Where d=double (gradient image)

$m n=\min$ (min (gradient image)), $m x=\max (\max$ (gradient image))

4. Define $3 \times 3$ square structuring elements [M] with set of fuzzy logic conditions.

5. Find fuzzy morphological gradient image $\mathrm{G}(\mathrm{r}): \mathrm{G}(\mathrm{r})$ $=(\mathrm{r} \oplus \mathrm{M})-(\mathrm{r} \ominus \mathrm{M})$ where $\mathrm{r}$ is Fuzzify gradient image and $\mathrm{M}$ is structuring element.

6. Apply gray thresholding to fuzzy morphological gradient image $\mathrm{G}(\mathrm{r})$ to get better edge.

\section{EXPERIMENT AND RESULT ANALYSIS}

In this section, the proposed fuzzy morphological gradient edge detection algorithm is compared with the existing morphological methodfor edge detection. Fig. 1 is the original rice image. Fig.2 is the result ofprocessed rice image after applying morphological gradient operator. Fig.3 and Fig.4 are the rice images processed by fuzzy morphological gradient operation and fuzzy morphological gradient thresholdingon detector .According to the experiment results, the 
fuzzymorphological gradient operation andfuzzy morphological gradient thresholding edge detector are successful in obtaining better edges in rice image and the detected edges are clearer than the edges detected by morphological detector.

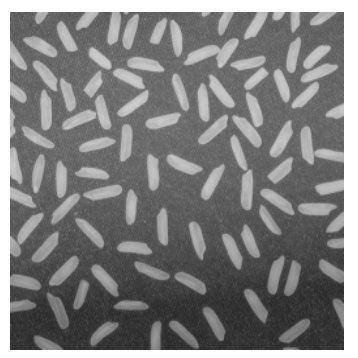

Figure 1: Original Rice Image

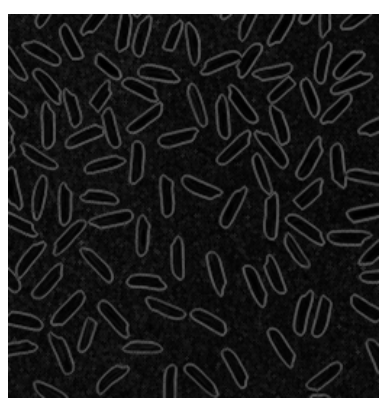

Figure 2: Morphological Edge

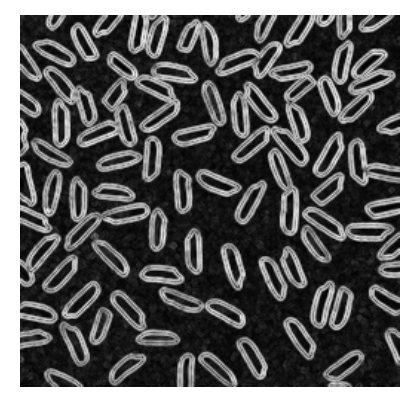

Figure3:Fuzzy Gradient Edge

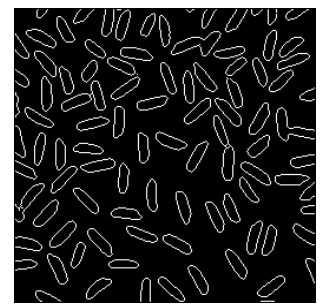

\section{CONCLUSION}

In this paper, a fuzzy morphological algorithm is proposed to detect edge for rice image. The experimental results show that the algorithm is more efficient for edge detection. Experimental results show that the proposed algorithm not only suppresses noise effectively, but also retains clear and continuous edges. Fuzzy morphology has the capability to work in environments of high tolerance and gives good results with remarkable accuracy. The purpose of this approach is to combine the advantages of fuzzy and sobel techniques.

\section{REFERENCES}

[1] R. Wang, L. Gao, S. Yang and Y. Liu, "An Edge detection method by combining fuzzy logic and Neural networks", Machine Learning and Cybernetics, vol.7, pp. 4539-4543, 18- 21, 2005.

[2] V. Mittal and M. S. Batra, "Edge Detection Technique by Using Nero Fuzzy System”, Proceedings of 2nd National Conference on Challenges \& Opportunities in Information Technology, RIMT-IET, Mandi Gobindgarh, 2008.

[3] R. Gonzalez, R. Woods and S. Eddins, "Digital Image Processing Using MATLAB”, Prentice Hall, 2004.

[4] A. Huertas, and G. Medioni, "Detection of intensity changes with sub pixel accuracy using Laplacian- Gaussian masks”, IEEE Trans. On Pattern Analysis and Machine Intelligence”, PAMI, vol. 8, pp. 651664,1986.

[5] J. Canny, “A Computational Approach to Edge Detection”, IEEE Trans, Pattern Anal.Mach. Intel, Vol.6, pp.679- 687, 1986.

[6] S D. Marr and E. Hildreth, "Theory of edge detection", Proc. Royal Soc., London, Vol. 207, pp. 187- 217, 1980.

[7] J. Musevi-Niya and A. Aghagolzadeh, "Adaptive directional waveletbased detection", 2nd International Symposium on Telecommunications (IST2003), Isfahan, Iran, pp.191- 195, 16- 18,2003.

[8] J.S.J Lee, R.M. Haralick, and L.G. Shapiro, "Morphological Edge Detection”, IEEE J. Robot. Automat,vol. 3, pp. 142-156, 1987.

[9] J. Serra, "Image Analysis and Mathematical Morphology", Academic Press, New York, 1982

[10] P. Maragos, "Differential Morphology and Image Processing", IEEE Trans Image Processing, vol. 5, pp. 922-937, 1996.

[11] A.P Richard, "A New Algorithm for Image Noise Reduction Using Mathematical morphology”, IEEE Transaction on Image Processing, vol. 4, Pp. 554-568, 1995.

[12] Rivest Jean, "Morphological Operators on Complex Signals", Signal Processing, vol. 84, pp. 133-139, 2004.

[13] Soille,"Morphological Image Analysis: Principles and Applications”, Springer, Berlin, 1999.

[14] Ehsan Nadarnejat, "Edge Detection Techniques: Evaluations and comparison”, Applied Mathematical Sciences, Vol 2, pp. 1507-152, 2008.

[15] D.R.Nayak, "Edge detection of images using fuzzy morphology", International Journal of Computer Application, vol.2, pp.52-57, 2012.

[16] Dr. H. B. Kekre, Ms. Saylee M. Gharge, "Image Segmentation using Extended Edge Operator for ammography Images,"International Journal on Computer Science and Engineering, Vol. 02, No. 04, 2010, 10861091.

[17] Yuqian Zhao, Weihua Gui and Zhencheng Chen, Tang Jing-tian and Li Ling-yun., "Medical Images Edge Detection Based on Mathematical Morphology", Proceedings of the IEEE Engineering in Medicine and Biology 27th Annual International Conference Shanghai, China, pp: 6492 -6495, 2005.

[18] Yuqian Zhao, Weihua Gui and Zhencheng Chen, "Edge Detection Based on Multi-Structure Elements Morphology", Proceedings of the 6th World Congress on Intelligent Control and Automation Dalian, China, vol.-2, pp: 9795 - 9798, 2006.

Figure 4: Fuzzy Morphological Thresholding Edge 


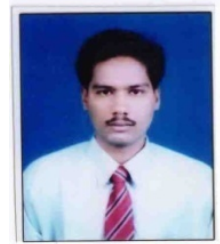

Dillip Ranjan Nayak received the B.Tech. (CSE) degree from North Orissa University. He completed his M.Tech. (computer Science)from NIT Rourkela. He worked as Asst.prof in C.V.Raman College of Engineering, Odisha for five years. Presently, he is working as Asst. Prof in Govt.College of Engineering kalahandi,under Biju Patnaik University, odisha, India. He has published 04 research articles. His research interests include Image Segmentation,Image Extracion and Medical Imaging. 